Thomas Wunder

\title{
Transnationale Strategien
}


GABLER EDITION WISSENSCHAFT 
Thomas Wunder

\section{Transnationale Strategien}

Anwendungsorientierte Realisierung mit Balanced Scorecards

Mit einem Geleitwort von Prof. Dr. Ulrich Grimm

Deutscher Universitäts-Verlag 
Bibliografische Information Der Deutschen Bibliothek Die Deutsche Bibliothek verzeichnet diese Publikation in der Deutschen Nationalbibliografie; detaillierte bibliografische Daten sind im Internet über $<$ http://dnb.ddb.de> abrufbar.

Dissertation European Business School Destrich-Winkel, 2003

\section{Auflage August 2004}

Alle Rechte vorbehalten

(C) Deutscher Universitäts-Verlag/GWV Fachverlage GmbH, Wiesbaden 2004

Lektorat: Ute Wrasmann / Britta Göhrisch-Radmacher

Der Deutsche Universitäts-Verlag ist ein Unternehmen von

Springer Science+Business Media.

www.duv.de

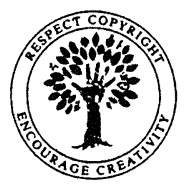

Das Werk einschließlich aller seiner Teile ist urheberrechtlich geschützt. Jede Verwertung außerhalb der engen Grenzen des Urheberrechtsgesetzes ist ohne Zustimmung des Verlags unzulässig und strafbar. Das gilt insbesondere für Vervielfältigungen, Übersetzungen, Mikroverfilmungen und die Einspeicherung und Verarbeitung in elektronischen Systemen.

Die Wiedergabe von Gebrauchsnamen, Handelsnamen, Warenbezeichnungen usw. in diesem Werk berechtigt auch ohne besondere Kennzeichnung nicht zu der Annahme, dass solche Namen im Sinne der Warenzeichen- und Markenschutz-Gesetzgebung als frei zu betrachten wären und daher von jedermann benutzt werden dürften.

Umschlaggestaltung: Regine Zimmer, Dipl.-Designerin, Frankfurt/Main

Gedruckt auf säurefreiem und chlorfrei gebleichtem Papier

ISBN-13:978-3-8244-0775-0

e-ISBN-13:978-3-322-81157-8

DOI: $10.1007 / 978-3-322-81157-8$ 


\section{Geleitwort}

Die Diskussion um das Spannungsfeld zwischen globaler Integration und lokaler Anpassung in international tätigen Unternehmen ist nicht neu. Mit der transnationalen Strategie hat hier ein hybrider Strategieansatz an Bedeutung gewonnen, durch den unterschiedliche Quellen von Wettbewerbsvorteilen simultan genutzt werden sollen. Zur Realisierung einer transnationalen Strategie skizzieren die bislang vorgelegten Konzepte meist das Ideal eines integrierten und hochvernetzten international tätigen Unternehmens, dem die Realität in vielen Fällen heute noch nicht entspricht. International tätige Unternehmen, die eine transnationale Strategie verfolgen und ihre Internationalität damit zur Basis von Wettbewerbsvorteilen machen wollen, verfügen nicht notwendigerweise auch über eine transnationale Organisationsstruktur in Form eines integrierten Netzwerkes. Es fehlen bislang schlüssige Konzepte, die diese Diskrepanz zwischen wissenschaftlichem Interesse und betrieblicher Realität überwinden und in der Unternehmenspraxis als Managementgrundlage für die Umsetzung der vielfältigen Anforderungen an die Realisierung einer transnationalen Strategie fungieren können.

Herr Wunder hat sich mit seiner Arbeit der verdienstvollen Aufgabe gestellt, dieses Defizit zu verringern. Dabei greift er mit der Balanced Scorecard (BSC) auf das derzeit prominenteste Konzept zur Strategieumsetzung zurück und überprüft dessen Leistungsfähigkeit für die Realisierung einer transnationalen Strategie. Als wesentliche zusätzliche Herausforderung im Vergleich zum üblichen Problemumfang stellt sich hierbei die Integration der länderspezifischen Besonderheiten in einem gemeinsamen Konzernrahmen dar.

Der Autor erreicht sein selbst gestecktes Ziel, indem er zunächst die vielfältigen strategischen, strukturellen und kulturellen Anforderungen an eine transnationale Strategie aus den dominierenden theoretischen Ansätzen extrahiert und systematisiert. Die Herausarbeitung des Problemlösungspotenzials der BSC ist nach meiner Auffassung das Glanzstück der Arbeit. Herr Wunder liefert hier eine derartig dichte und klare Zusammenstellung sowie kritische Auseinandersetzung mit den BSC-Konzeptmerkmalen, wie es sie nach meinem Kenntnisstand bislang noch nicht gab. Die gelungene Gegenüberstellung der Anforderungskriterien mit dem Problemlösungspotenzial der BSC wird durch zwei anschauliche Praxisbeispiele illustriert und bewertet. Zur Überprüfung der Praxistauglichkeit verwendet Herr Wunder einen umfangreichen Fragebogen für Experteninterviews mit Managern aus der Zentrale und den internationalen Tochtergesellschaften. 
Das vorliegende Buch richtet sich vornehmlich an Manager in international tätigen Unternehmen, sowie Verantwortliche für strategische Unternehmensplanung und Konzernentwicklung. Vor dem Hintergrund des Spannungsfeldes zwischen globaler Integration und lokaler Anpassung finden sie innovative und praxisorientierte Hinweise zum Einsatz von Balanced Scorecards in internationalen Konzernstrukturen. Der besondere Wert für den Leser liegt dabei nicht nur in dem vorgestellten methodischen Fortschritt, sondern auch in den leicht nachvollziehbaren Anwendungsbeschreibungen, die um konkrete Empfehlungen aus den gesammelten Praxiserfahrungen ergänzt wurden. Aufgrund seines Innovationsgrades kann ich die Lektüre der vorliegenden Arbeit auch für Dozenten und Studierende der Betriebswirtschaftslehre sehr empfehlen.

Prof. Dr. Ulrich Grimm 


\section{Vorwort}

Ausschlaggebend für die Wahl meines Dissertationsthemas waren persönliche Erfahrungen in international tätigen Unternehmen, wo ich als Managementberater immer wieder mit Problemstellungen aus dem Spannungsfeld zwischen globaler Integration und lokalen Anforderungen in Berührung kam. Im Mittelpunkt stand dabei immer die Nutzbarmachung des in der „Internationalität" dieser Unternehmen liegenden Potenzials für die Realisierung von Wettbewerbsvorteilen. Wiederholt stellte sich die Frage, wie eine optimale Balance zwischen den strategischen Konzernanforderungen einerseits und den lokalen Fliehkräften internationaler Tochtergesellschaften andererseits geschaffen werden kann. Der in meiner Arbeit fokussierte Ansatz der transnationalen Strategie liefert hierfür einen geeigneten Bezugspunkt. Kritische Stimmen in Wissenschaft und Praxis zu den meist idealtypischen Empfehlungen für die Realisierung einer transnationalen Strategie aus den hierfür dominierenden Managementmodellen haben mich in meinem Vorhaben bestätigt, das Problemlösungspotenzial des Balanced Scorecard Ansatzes für diese spezifische Herausforderung wissenschaftlich zu untersuchen.

Im Rahmen meiner Dissertation war es für mich vor allem wichtig, eine Brücke zwischen der Theoriewelt und der Unternehmensrealität zu bauen. Die ständige Interaktion mit meinen Praxispartnern im Rahmen der gewählten Aktionsforschung sowie die regelmäßigen Forschungskolloquien an der European Business School (ebs) und die inspirierenden Diskussionen am Lehrstuhl für Strategisches Management stellten sich für mich als wichtige Impuls- und Motivationsfaktoren heraus. Dabei war für mich insbesondere die kritische Auseinandersetzung mit den Fragen der „Wissenschaftlichkeit“ und der generellen „Steuerbarkeit sozialer Systeme" sehr bereichernd. In den Jahren meiner Forschungstätigkeit bin ich zu der Überzeugung gekommen, dass die Maßstäbe dessen, was als „wissenschaftlich“ anerkannt wird, in hohem Maße von den zeit-, raum- und disziplinabhängigen Forschungsparadigmen bestimmt sind und es hierfür keine abschließende Antwort gibt. Mit der von mir gewählten Herangehensweise einer engen Verzahnung von Wissenschaft und Praxis während des gesamten Forschungsprozesses konnte ich nicht nur einen persönlichen Anspruch erfüllen, es hat sich in den vergangenen Jahren auch immer wieder bestätigt, dass mein gewähltes Thema für die Unternehmen von großer Bedeutung ist.

Den Entstehungsprozess der vorliegenden Arbeit haben viele ermöglicht, begleitet und gefördert, denen ich an dieser Stelle Dank sagen möchte. An erster Stelle ist hier mein akademischer Betreuer, Herr Prof. Dr. Ulrich Grimm, zu nennen, den ich als Doktorvater sehr schätzen gelernt habe. Die Zusammenarbeit mit ihm war und ist für mich sowohl wissenschaftlich als auch persönlich prägend und dafür gilt ihm mein herzlicher Dank. Herrn Prof. Dr. JeanPaul Thommen bin ich sehr verbunden, weil er mir als Zweitgutachter durch seine konstruktiven Anmerkungen und Fragen während der Doktorandenkolloquien neue Perspektiven aufgezeigt und damit das Gelingen der Arbeit in hohem Maße gefördert hat. Herrn Prof. Dr. Utz 
Schäffer, Herrn Prof. Dr. Hartmut Kreikebaum und Herrn Prof. Dr. Jürgen Bunge danke ich für das Interesse an meiner Arbeit und die konstruktiven Fragen während meiner Disputation. Ein herzlicher Dank geht auch an Herrn Dr. Matthias Baur für die vielen hilfreichen Impulse.

Eine berufsbegleitende Promotion als Mitarbeiter einer internationalen Managementberatung ist nur mit ausreichend zeitlichen und gedanklichen Freiräumen möglich. Hervorheben möchte ich zunächst Herrn Prof. Dr. Péter Horváth. Er verkörpert als Firmengründer und -mitgestalter von Horváth \& Partners in beispielhafter Form die Verzahnung von Wissenschaft und Praxis, die ich auch als Anspruch für meine eigene Forschungstätigkeit betrachtet habe. Stellvertretend für das gesamte Führungsteam von Horváth \& Partners sei an dieser Stelle dem Sprecher des Vorstandes der Horváth AG, Herrn Dr. Bernd Gaiser, für die vielen wichtigen Impulse in gemeinsamen Projekten sowie die Möglichkeit und Förderung der berufsbegleitenden und praxisnahen Promotion gedankt. Meiner damaligen Mentorin, Frau Dr. Carmen Andrea Fink, danke ich für den notwendigen Freiraum und Ansporn, ohne den diese Arbeit nicht möglich gewesen wäre. Ein spezieller und freundschaftlicher Dank gebührt Herrn Dr. Thomas Schulz und Herrn Dr. Michael Currle für die fortwährende Unterstützung meines Promotionsprozesses.

Als weiteres ist die große Offenheit und Bereitschaft meiner beiden Praxispartner hervorzuheben, ohne deren Hilfe meine praxisnahe Forschungsarbeit nicht möglich gewesen wäre. Dafür bedanke ich mich bei den verantwortlichen Führungskräften, die mir die Beschreibung und Bewertung der in ihren Unternehmen gemachten Erfahrungen ermöglicht haben. Zu betonen ist hier insbesondere die Gelegenheit, mit wesentlichen Teilen des internationalen Top Managements sehr zeitintensive Experteninterviews zu führen, kritisch auszuwerten und in Form von Praxisbeispielen darzustellen. Ein herzlicher Dank gilt hierbei auch den zahlreichen Interviewpartnern für die offenen und konstruktiven Gespräche. Aus Gründen der Anonymität verzichte ich an dieser Stelle auf eine namentliche Nennung.

Bei der Fertigstellung der Arbeit und den Korrekturen hat mich besonders Herr Michael Rank unterstützt. Die Veröffentlichung im DUV-Verlag begleiteten Frau Ute Wrasmann und Frau Britta Göhrisch-Radmacher. Auch ihnen bin ich zu Dank verpflichtet.

Zum guten Schluss gebührt der größte Dank meinem Vater, Herrn Fritz Wunder, der in beispielhafter Weise die Grundlage für meine Entwicklung geschaffen und mich in jeder Hinsicht unterstützt hat. Herzlichen Dank auch an meine eng verbundene Freundin, Frau Jeannine Subra, die mich über viele Jahre immer wieder zu diesem Schritt ermuntert hat. 


\section{Inhaltsübersicht}

Inhaltsverzeichnis XI

Abbildungsverzeichnis XVII

Abkürzungsverzeichnis. XIX

1 Einführung 1

2 Terminologischer und konzeptioneller Bezugsrahmen 19

3 Potenzial der Balanced Scorecard für die Realisierung einer transnationalen Strategie 84

4 Praxisbeispiele - Illustration und Bewertung. 209

5 Zusammenfassung, Anwendungsimplikationen und Ausblick 263

Anhang I: Empirische Studien zur Balanced Scorecard. 271

Anhang II: Interviewleitfaden 273

Literaturverzeichnis .289 


\section{Inhaltsverzeichnis}

Inhaltsübersicht ................................................................................................................ IX

Inhaltsverzeichnis .......................................................................................................... XI

Abbildungsverzeichnis ..........................................................................................XVII

Abkürzungsverzeichnis ........................................................................................... XIX

1 Einführung...........................................................................................................................

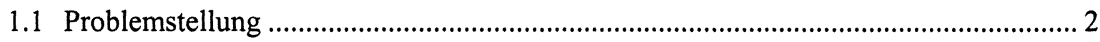

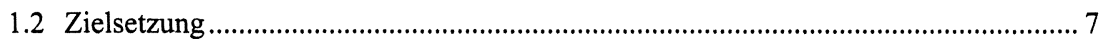

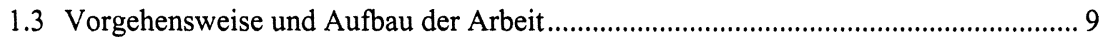

1.4 Relevanz des Themas und Innovationsbeitrag....................................................... 11

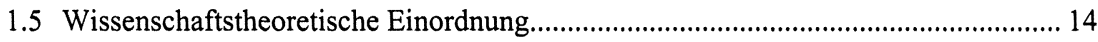

1.5.1 Forschungsobjekt und Schwerpunktbildung.................................................. 15

1.5.2 Forschungsmethodik ................................................................................. 16

2 Terminologischer und konzeptioneller Bezugsrahmen................................19

2.1 Strategie und Strategierealisierung ........................................................................ 19

2.1.1 Grundlegendes Strategieverständnis....................................................... 19

2.1.1.1 Ursprung und Sichtweisen des Strategiebegriffs.............................. 19

2.1.1.2 Merkmale des zugrunde gelegten Strategieverständnisses................ 23

2.1.1.2.1 Zweck-Mittel-Relation ................................................... 23

2.1.1.2.2 Erfolgspotenziale und Wettbewerbsvorteile..................... 26

2.1.1.2.3 Integration unterschiedlicher Strategiesichten................. 28

2.1.1.2.4 Pluralität strategischer Stoßrichtungen............................ 30

2.1.1.3 Konsolidierung und Eingrenzung................................................... 31

2.1.2 Grundlegendes Strategierealisierungsverständnis ........................................... 36

2.1.2.1 Ursprung und Sichtweisen von Strategieprozessen ............................ 36

2.1.2.2 Merkmale des zugrunde gelegten

Strategierealisierungsverständnisses .............................................. 39

2.1.2.2.1 Konvergenz von Formulierung und Implementierung .... 39

2.1.2.2.2 Ganzheitliche Betrachtung: Strategie-Struktur-Kultur... 41

2.1.2.2.3 Dynamisch-iterative Betrachtung ................................... 42

2.1.2.2.4 Offenheit der Strategieentstehung ................................... 43

2.1.2.3 Konsolidierung und Eingrenzung................................................... 44

2.2 Transnationale Strategie.......................................................................................... 47 
2.2.1 Wettbewerbsvorteile im Spannungsfeld ,glokaler“ Anforderungen ................ 48

2.2.1.1 Die Dimension ,globale Integration“........................................... 50

2.2.1.2 Die Dimension ,lokale Anpassung“............................................... 51

2.2.2 Strategische Ansätze im Spannungsfeld ,glokaler“ Anforderungen............... 52

2.2.2.1 Kontingenztheoretische Ansätze .................................................. 52

2.2.2.1.1 Matrixgestützte Kontingenzansätze............................... 53

2.2.2.1.2 Koordination - Konfiguration: Wertkettenansatz ........... 55

2.2.2.2 Idealtypische Strategiealternativen international tätiger

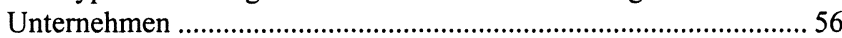

2.2.3 Herleitung eines erweiterten Verständnisses zum Spannungsfeld ,globale Integration“ und ,lokale Anpassung “........................................................... 59

2.2.3.1 Neuere organisationstheoretische Überlegungen ............................. 59

2.2.3.1.1 Defizite matrixgestützter Kontingenzansätze................. 59

2.2.3.1.2 Das Spannungsfeld im Lichte der Systemtheorie............ 61

2.2.3.1.3 Das Spannungsfeld im Lichte der postmodernen Organisationstheorie ..................................................... 63

2.2.3.2 Neuere praxeologische Ansätze......................................................64 64

2.2.4 „Transnationale Strategie“ als Ausdruck eines neuen Paradigmas international tätiger Unternernehmen? 67

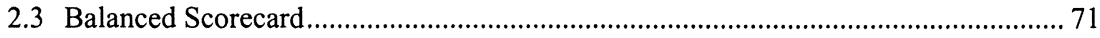

2.3.1 Ursprung und Entwicklung der Balanced Scorecard ................................... 74

2.3.2 Betrachtungsebenen des Balanced Scorecard-Konzeptes ............................... 77

2.3.2.1 Die inhaltliche Ebene des Balanced Scorecard-Konzeptes .............. 78

2.3.2.2 Die prozessuale Ebene des Balanced Scorecard-Konzeptes ............ 80

2.3.3 Konsolidierung und Eingrenzung ............................................................... 82

\section{Potenzial der Balanced Scorecard für die Realisierung einer} transnationalen Strategie

3.1 Anforderungen für die Realisierung einer transnationalen Strategie......................... 84

3.1.1 Managementkonzepte zur Realisierung einer transnationalen Strategie ......... 84

3.1.1.1 Ansatz der ,unternehmensspezifischen Managementqualität" ........ 85

3.1.1.2 Ansatz der ,transnationalen Wettbewerbsfähigkeit" ........................ 89

3.1.1.3 Ansatz des , ,heterarchischen Managements“.................................. 95

3.1.1.4 Ansatz der , horizontalen Organisation“.......................................... 98

3.1.1.5 Ansatz des ,globalen Netzwerkmanagements“............................. 100

3.1.2 Ableitung wesentlicher übergreifender Merkmale ...................................... 102

3.1.2.1 Strategische Merkmale ................................................................ 104

3.1.2.2 Strukturelle Merkmale ............................................................... 106

3.1.2.3 Kulturelle Merkmale................................................................... 108

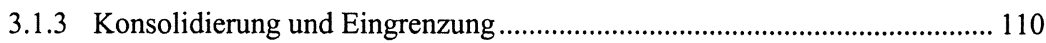


3.2 Konzeptmerkmale und Problemlösungspotenzial der BSC ........................................ 113

3.2.1 Inhaltliche Konzeptmerkmale der Balanced Scorecard................................... 115

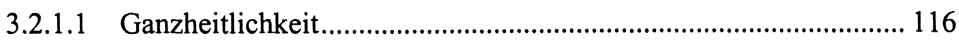

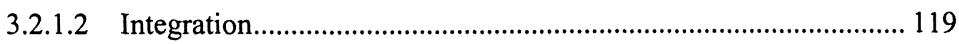

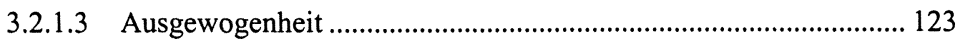

3.2.1.4 Zwischenbetrachtung: Polytelie und Zielinstrumentalität............... 125

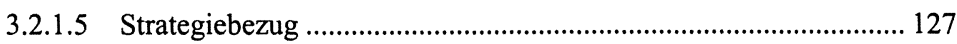

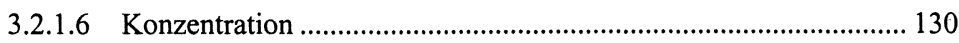

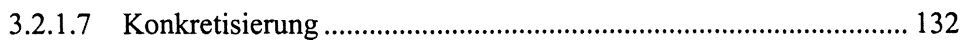

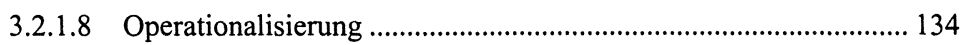

3.2.1.9 Zwischenbetrachtung: Fokussierung, Spezifität und Anforderungsgehalt ..................................................................... 136

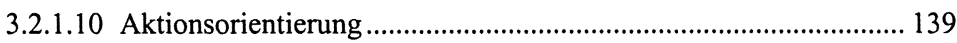

3.2.2 Prozessuale Konzeptmerkmale der Balanced Scorecard ................................ 140

3.2.2.1 Strukturierung der Zielfindung..................................................... 140

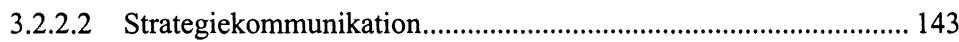

3.2.2.3 Integration von Managementsystemen .......................................... 145

3.2.2.4 Organisationales Lernen .............................................................. 148

3.2.2.5 Strategiedynamisierung und -adaption ......................................... 149

3.2.3 Abschließende Würdigung des Balanced Scorecard-Konzeptes.................... 153

3.3 Gegenüberstellung von Anforderungen und Konzeptmerkmalen .............................. 157

3.3.1 Strategische Anforderungen und Balanced Scorecard ................................... 157

3.3.1.1 Simultane Nutzung von globalen Skaleneffekten, Verbundvorteilen und Vorteilen aus lokalen Unterschieden.......... 157

3.3.1.1.1 Globale statische und dynamische Skaleneffekte.......... 160

3.3.1.1.2 Globale Verbundvorteile .............................................. 164

3.3.1.1.3 Vorteile bzw. Notwendigkeiten einer lokalen Anpassung ……………………………………………... 165

3.3.1.2 Kontinuierliche Suche nach globalen Lernpotenzialen und

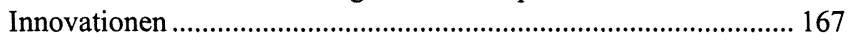

3.3.1.2.1 Erweiterung von organisationalen Wissensformen ....... 169

3.3.1.2.2 Umwandlung von organisationalen Wissensformen ..... 172

3.3.1.3 Permanente Nutzung geografischer

Risikodiversifikationsmöglichkeiten ............................................ 173

3.3.1.4 Konsolidierung und Zwischenbewertung..................................... 175

3.3.2 Strukturelle Anforderungen und Balanced Scorecard ................................... 178

3.3.2.1 Optimierung der globalen Konfiguration interdependenter

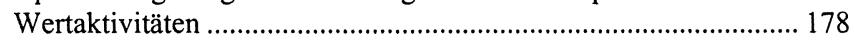

3.3.2.2 Unterdeterminiertheit und flexible Dominanz von

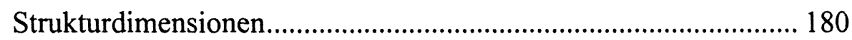

3.3.2.2.1 Rolle der strategischen Führung ...................................... 181 
3.3.2.2.2 Rolle der strategischen Unterstützung............................. 183

3.3.2.2.3 Rolle der strategischen Umsetzung ............................... 183

3.3.2.2.4 Rolle des „schwarzen Lochs“........................................ 184

3.3.2.3 Differenzierte Prüfung der Vorteilspotenziale von

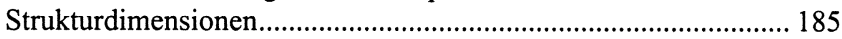

3.3.2.4 Konsolidierung und Zwischenbewertung ..................................... 186

3.3.3 Kulturelle Anforderungen und Balanced Scorecard...................................... 189

3.3.3.1 Sicherstellung einer geozentrisch-holistischen

Verhaltensorientierung ............................................................... 190

3.3.3.1.1 Eigenlogiken ............................................................... 191

3.3.3.1.2 Eigeninteressen ............................................................... 193

3.3.3.2 Verankerung gemeinsamer inter-kultureller Orientierungspunkte. 196

3.3.3.3 Etablieren einer offenen Informations-, Kommunikations- und

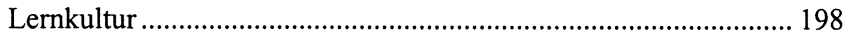

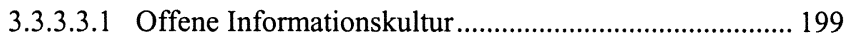

3.3.3.3.2 Offene Kommunikationskultur......................................... 200

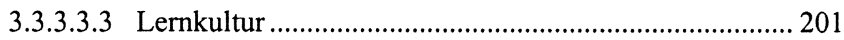

3.3.3.4 Konsolidierung und Zwischenbewertung..................................... 202

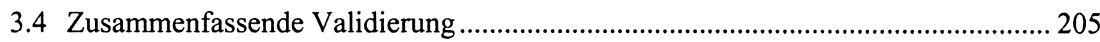

4 Praxisbeispiele - Illustration und Bewertung .........................................209

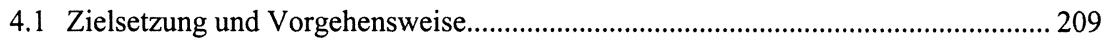

4.2 Praxisbeispiel 1: Oilcomp - Geschäftsbereich EP ……………………………….... 213

4.2.1 Unternehmensprofil, Ausgangssituation und Zielsetzung............................. 213

4.2.2 Beschreibung von Vorgehensweise und Ergebnissen .................................. 215

4.2.2.1 Balanced Scorecard-Entwicklung für EP ..................................... 215

4.2.2.2 Globale Kaskadierung der EP Balanced Scorecard......................... 217

4.2.2.3 Synchronisation und Abstimmung der Balanced Scorecards......... 220

4.2.2.4 Verankerung des Balanced Scorecard-Systems ............................. 222

4.2.2.5 BSC-Review und Anpassung der BSC-Architektur......................... 223

4.2.3 Realisierter Anwendungsstand und Ausblick............................................... 225

4.2.4 Lösungsbewertung im Hinblick auf transnationale Strategieelemente ......... 226

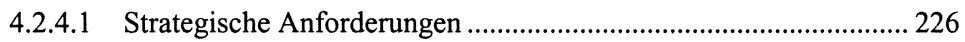

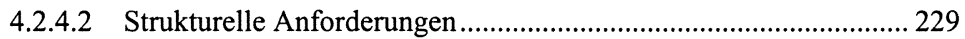

4.2.4.3 Kulturelle Anforderungen........................................................... 231

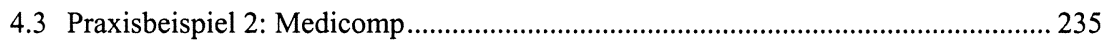

4.3.1 Unternehmensprofil, Ausgangssituation und Zielsetzung.............................. 235

4.3.2 Beschreibung der Vorgehensweise und Ergebnisse ..................................... 236

4.3.2.1 Balanced Scorecard-Entwicklung für Medicomp............................ 236 
4.3.2.2 Globale Kaskadierung der Medicomp-BSC

237

4.3.2.3 Synchronisation und Abstimmung der Balanced Scorecards......... 239

4.3.2.4 Verankerung des Balanced Scorecard-Systems ............................ 240

4.3.2.5 BSC-Review und Anpassung der BSC-Architektur....................... 241

4.3.3 Realisierter Anwendungsstand und Ausblick ............................................. 241

4.3.4 Lösungsbewertung im Hinblick auf transnationale Strategieelemente ......... 242

4.3.4.1 Strategische Anforderungen ........................................................ 242

4.3.4.2 Strukturelle Anforderungen ..................................................... 247

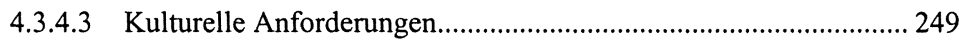

4.4 Zusammenfassende Validierung der beiden Praxisanwendungen ........................... 257

4.5 Abschließende Überlegungen zur Allgemeingültigkeit der Aussagen ..................... 260

5 Zusammenfassung, Anwendungsimplikationen und Ausblick ................263

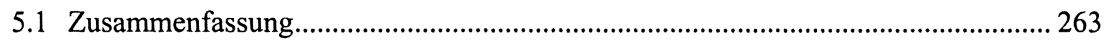

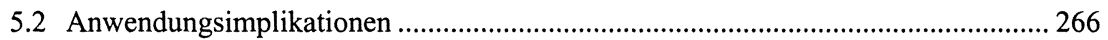

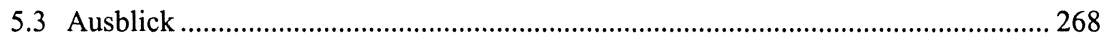

Anhang I: Empirische Studien zur Balanced Scorecard ................................271

Anhang II: Interviewleitfaden ......................................................................273

Literaturverzeichnis .....................................................................................289 


\section{Abbildungsverzeichnis}

Abb. 1-1: Rahmenbedingungen international tätiger Unternehmen ................................. 3

Abb. 1-2: Strategischer Anspruch des international tätigen Unternehmens ....................... 5

Abb. 1-3: Gedankenflussplan der Arbeit........................................................................ 11

Abb. 2-1: Grundmuster von empirisch beobachtbaren Strategien nach Mintzberg ............ 22

Abb. 2-2: Wirkungsweisen und Komponenten einer Vision ............................................. 25

Abb. 2-3: Grundverständnis von Strategie als konsistentes Handlungsmuster zur Vision. 26

Abb. 2-4: Strategisches Dreieck als Bezugsrahmen für Wettbewerbsvorteile.................... 27

Abb. 2-5: Klassifikation von strategischen Handlungen................................................. 33

Abb. 2-6: Betrachtungsbereich im Rahmen des zugrunde gelegten

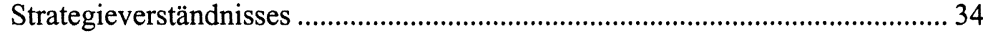

Abb. 2 -7: Charakterisierung des zugrundegelegten Strategieverständnisses ..................... 35

Abb. 2-8: Denkschulen des strategischen Managements .................................................. 37

Abb. 2-9: Konvergenz von Strategieformulierung und -implementierung ......................... 39

Abb. 2-10: Holistische Betrachtungsweise in der Trilogie Strategie-Struktur-Kultur......... 42

Abb. 2-11: Idealtypische Arten von Internationalisierungsstrategien ................................ 57

Abb. 2-12: Quellen von Wettbewerbsvorteilen in international tätigen Unternehmen......... 66

Abb. 2-13: Transnationale Strategie als „Meta-Perspektive“ des

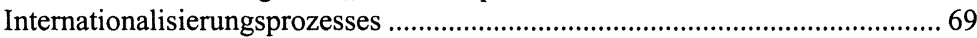

Abb. 2-14: Entwicklungsstufen des Balanced Scorecard-Konzeptes................................... 76

Abb. 2-15: Inhaltskomponenten einer Balanced Scorecard ............................................... 78

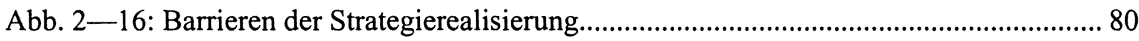

Abb. 2-17: Die Balanced Scorecard als strategischer Handlungsrahmen ............................. 82

Abb. 3-1: Multinationale, globale, internationale und transnationale Strategietypologien

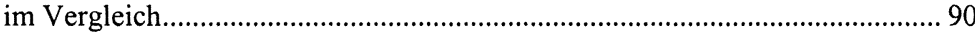

Abb. 3-2: Strategietypologien international tätiger Unternehmen .................................... 91

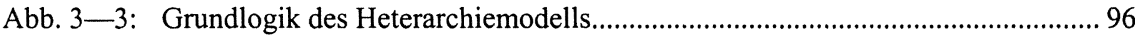

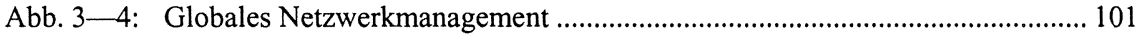

Abb. 3-5: Erweiterung des Bezugsrahmens von globaler Integration und lokaler

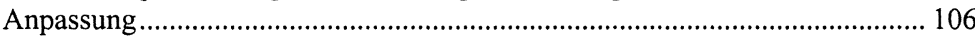

Abb. 3-6: Anforderungen für die Realisierung einer transnationalen Strategie................ 112

Abb. 3-7: Bedingungen des Verhaltens........................................................................... 114

Abb. 3-8: Inhaltliche und prozessuale Konzeptmerkmale der Balanced Scorecard ......... 115

Abb. 3-9: Grundstruktur des Zielsystems „Balanced Scorecard“.................................... 116

Abb. 3-10: Zusammenführung unterschiedlicher Strategiesichten in der Balanced 
Abb. 3-11: Praxisbeispiel einer Strategy Map (modifiziert) 120

Abb. 3-12: Ausgewogenheit als Konzeptmerkmal der Balanced Scorecard

Abb. 3-13: Integration geplanter und emergenter Strategieprozesse im Balanced Scorecard-System......

Abb. 3-14: Gesamtmodell der Zielwirkungen

Abb. 3-15: Grundwirkungsweise der Balanced Scorecard im Rahmen der Strategierealisierung.

Abb. 3-16: BSC als Basis für Vorteile aus Skalen- und Verbundeffekten sowie lokalen Unterschieden. 158

Abb. 3-17: Organisationales Lernen mit Balanced Scorecard 169

Abb. 3-18: BSC-Entwicklung als Basis für den Aufbau von explizitem strategierelevantem Wissen.

Abb. 3-19: BSC-Architektur im Spannungsfeld verschiedener Vorteilsquellen 176

Abb. 3-20: BSC-Kaskadierungsmethoden und Rollen internationaler Tochtergesellschaften.

Abb. 3-21: Individuelle Prüfung von Vorteilspotenzialen auf der Basis von Balanced Scorecards

Abb. 3-22: BSC-Kaskadierung in einer globalen Matrix-Struktur. 188

Abb. 3-23: Eigenlogiken und Eigeninteressen als Anknüpfungspunkte der Balanced Scorecard.

Abb. 3-24: Bezugsrahmen für Aufbau und Anwendung eines Balanced ScorecardSystems 207

Abb. 4-1: Geschäftsbereichsorganisation des Oilcomp Konzerns 214

Abb. 4-2: Ergebniskomponenten der BSC-Entwicklung für Oilcomp EP. 217

Abb. 4 -3: Ursprüngliche Balanced Scorecard-Architektur bei Oilcomp EP. 218

Abb. 4 - 4: Kaskadierung mit eigenständiger BSC-Entwicklung 219

Abb. 4-5: Klärung und Darstellung der strategischen Kernaussagen einer Strategy Map 220

Abb. 4-6: Vorlage für die inhaltliche Abstimmung der Balanced Scorecards. 222

Abb. 4-7: MbO-Ziel zur BSC-Verankerung in internationalen Tochtergesellschaften .... 223

Abb. 4 -8: Strategische Anforderungen für den BSC-Review in internationalen Tochtergesellschaften.

Abb. 4-9: Balanced Scorecard-Architektur für die Pilotierung bei Medicomp 238

Abb. 4-10: Balanced Scorecard-Struktur für Tochtergesellschaften mit fixen und flexiblen Bereichen

Abb. 4-11: Fallstudienvergleich bezüglich der Realisierung einer transnationalen Strategie

Abb. 4-12: Innovative Action Research Cycle 


\section{Abkürzungsverzeichnis}

\begin{tabular}{|c|c|c|c|}
\hline A.d. V. & $\begin{array}{l}\text { Anmerkung des } \\
\text { Verfassers }\end{array}$ & HBS & Harvard Business School \\
\hline Abb. & Abbildung & HNO & Hals, Nasen, Ohren \\
\hline Aufl. & Auflage & Hrsg. & Herausgeber \\
\hline $\mathrm{Bd}$. & Band & HSEQ & Health, Safety, \\
\hline BFuP & $\begin{array}{l}\text { Betriebswirtschaft in } \\
\text { Forschung und Praxis }\end{array}$ & i. d. R. & $\begin{array}{l}\text { Environment, Quality } \\
\text { in der Regel }\end{array}$ \\
\hline boe & Barrel Öläquivalent & i. e. S. & im engeren Sinne \\
\hline $\mathrm{BSC}$ & Balanced Scorecard & i. S. & im Sinne \\
\hline bspw. & beispielsweise & i. S.e. & im Sinnes eines \\
\hline bzw. & beziehungsweise & i. w. S. & im weiteren Sinne \\
\hline $\mathrm{CCM}$ & $\begin{array}{l}\text { Center for Controlling and } \\
\text { Management }\end{array}$ & $\begin{array}{l}\text { Inc. } \\
\text { Int. }\end{array}$ & $\begin{array}{l}\text { Incorporated } \\
\text { International }\end{array}$ \\
\hline d. h. & das heißt & IT & Informationstechnologie \\
\hline DBW & $\begin{array}{l}\text { Die Betriebswirtschaft } \\
\text { (Zeitschrift) }\end{array}$ & $\begin{array}{l}\text { IR } \\
\text { Jg. }\end{array}$ & $\begin{array}{l}\text { Integration-Responsiveness } \\
\text { Jahrgang }\end{array}$ \\
\hline Diss. & Dissertation & Kap. & Kapitel \\
\hline DMNC & $\begin{array}{l}\text { Diversified (Multibusiness) } \\
\text { Multinational Corporations }\end{array}$ & KonTrag & $\begin{array}{l}\text { Kontrolle und Transparenz } \\
\text { im Unternehmensbereich }\end{array}$ \\
\hline $\begin{array}{l}\text { e. V. } \\
\text { EFQM }\end{array}$ & $\begin{array}{l}\text { eingetragener Verein } \\
\text { European Foundation for }\end{array}$ & $\mathrm{krp}$ & $\begin{array}{l}\text { Kostenrechnungs-Praxis } \\
\text { (Zeitschrift) }\end{array}$ \\
\hline & Quality Management & $\mathrm{MbO}$ & Management by Objectives \\
\hline EP & $\begin{array}{l}\text { Exporation \& Produktion } \\
\text { (Geschäftsbereich) }\end{array}$ & $\operatorname{mir}$ & $\begin{array}{l}\text { Management Inter- } \\
\text { national Review }\end{array}$ \\
\hline EPG & $\begin{array}{l}\text { ethnocentric, polycentric, } \\
\text { geocentric }\end{array}$ & MIS & $\begin{array}{l}\text { (Zeitschrift) } \\
\text { Management }\end{array}$ \\
\hline EPRG & $\begin{array}{l}\text { ethnocentric, polycentric, } \\
\text { regiocentric, geocentric }\end{array}$ & MNC & $\begin{array}{l}\text { Informationssysteme } \\
\text { Multinational Corporation }\end{array}$ \\
\hline ERP & $\begin{array}{l}\text { Enterprise Resource Plan- } \\
\text { ning }\end{array}$ & $\begin{array}{l}\text { NGL } \\
\text { Nr. }\end{array}$ & $\begin{array}{l}\text { Natural Gas Liquids } \\
\text { Nummer }\end{array}$ \\
\hline et al. & und andere & o. Jg. & ohne Jahrgang \\
\hline etc. & und so weiter & o. V. & ohne Verfasser \\
\hline evtl. & eventuell & o. Nr. & ohne Nummer \\
\hline F\&E & $\begin{array}{l}\text { Forschung und } \\
\text { Entwicklung } \\
\text { fortfolgende }\end{array}$ & OECD & $\begin{array}{l}\text { Organization for } \\
\text { Economic Cooperation and }\end{array}$ \\
\hline ff. & $\begin{array}{l}\text { fortfolgende } \\
\text { folgende }\end{array}$ & & Development \\
\hline $\begin{array}{l}\text { f. } \\
\text { ggf. }\end{array}$ & $\begin{array}{l}\text { folgende } \\
\text { gegebenenfalls }\end{array}$ & OEM & $\begin{array}{l}\text { Original Equipment Manu- } \\
\text { facturer }\end{array}$ \\
\hline $\begin{array}{l}\text { ggf. } \\
\text { GM }\end{array}$ & General Manager & $R \& D$ & Research \& Development \\
\hline H.O. & Head Office & S. & Seite \\
\hline HBR & Harvard Business Review & Sp. & Spalte \\
\hline
\end{tabular}


TNC Transnational

Corporation

u. a. unter anderem

u. U. unter Umständen

UK United Kingdom

UNCTAD United National

Conference on Trade and

Development

US United States

USA United States of America

US-GAAP US-Generally Accepted

Accounting Standards

US-\$ US-Dollar

vgl. vergleiche

Vol. Volume

v. von

vs. versus

WiSt Wirtschaftswissenschaftliches Studium

(Zeitschrift)

WISU Das Wirtschaftsstudium

(Zeitschrift)

z. B. zum Beispiel

ZfB Zeitschrift für Betriebswirtschaft

ZfO Zeitschrift für Organisation

ZfbF Zeitschrift für betriebswirtschaftliche Forschung

ZP Zeitschrift für Planung 